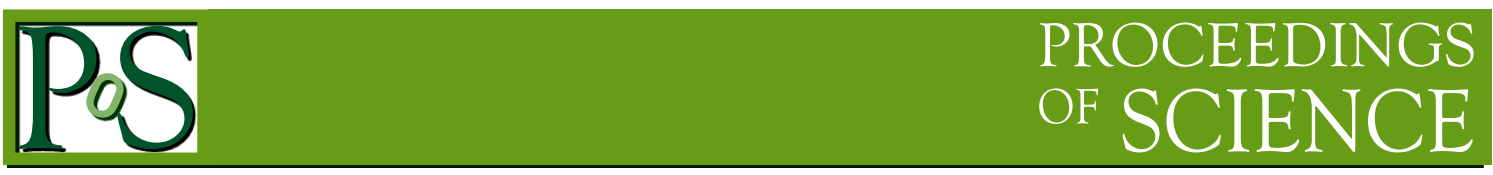

\title{
Status of the Polarized Molecular Source
}

\section{K. Toporkov\# ${ }^{1}$, D.M. Nikolenko, I.A. Rachek, Yu.V. Shestakov\# and A.V. Yurchenko\#}

Budker Institute of Nuclear Physics, 630090 Novosibirsk, Russia

\# also at Novosibirsk State University, 630090 Novosibirsk, Russia

E-mail: D.K.Toporkov@inp.nsk.su

\section{R. Engels and L. Huxold\#}

Institute for Nuclear Physics, Forschungszentrum Jülich, 52428 Jülich, Germany

\# also at Heinrich-Heine University Düsseldorf, Institute for Laser-and Plasma Physics, 40225

Düsseldorf, Germany

E-mail: $\underline{\text { r.w.engels } @ f z-j u e l i c h . d e}$

\section{Büscher}

Heinrich-Heine University Düsseldorf, Institute for Laser- and Plasma Physics, 40225

Düsseldorf, Germany

also at Peter Grünberg Institute (PGI-6), Forschungszentrum Jülich, 52428 Jülich, Germany

E-mail: Markus.Buescher@uni-duesseldorf.de

A novel source of polarized hydrogen and deuterium molecules has been tested. The use of sextupole superconducting magnets allows us to select molecules with the nuclear spin projection -1 for hydrogen and -2 for deuterium. The measured beam intensity of polarized hydrogen molecules for the nozzle temperature range of $6.5-30 \mathrm{~K}$ and a gas flow rate up to $6.6 \cdot 10^{-2} \mathrm{mbar} \cdot 1 / \mathrm{s}$ is presented. The measured flux of polarized hydrogen molecules of $\sim 3 \cdot 10^{12} \mathrm{~mol} / \mathrm{s}$ is in reasonable agreement with the Monte-Carlo simulation. The obtained results can be used as a basis for the development of a high-intensity source of polarized molecules.

23rd International Spin Physics Symposium - SPIN2018 -

10-14 September, 2018

Ferrara, Italy

\section{${ }^{1}$ Speaker}




\section{Introduction}

The sources of polarized particles are widely used for the production of polarized highenergy ion beams and polarized gas targets internal to storage rings of charged particles $[1,2]$. An additional interest in polarized sources is connected with the idea of using polarized fuel for the future thermonuclear fusion. Publications discussing the possibility of using this fuel in nuclear fusion reactions for energy production appear in scientific literature [3] more and more frequently. It is expected that the use of the polarized fuel would significantly increase the energy yield in D-T and ${ }^{3} \mathrm{He}-\mathrm{D}$ reactions. The effect of the polarization of particles involved in the reaction was confirmed in an experiment. If both particles are polarized, an enhancement of the fusion yield close to a factor of 1.5 is expected [4]. The double polarized D-D reactions are being experimentally studied at present [3]. The polarized helium atoms can be stored for a long time (hours) at a relatively high pressure (10 atmospheres and higher). Thus, the technological state of production of ${ }^{3} \mathrm{He}$, in principle, satisfies the requirements of reactors with polarized fuel even now. The modern atomic beam sources (ABSs) of highly polarized hydrogen and deuterium atoms have reached a limit of $\approx 10^{17}$ atoms/s. The produced atoms are also extremely reactive radicals, which does not allow one to accumulate and store them for a long time.

The research to obtain polarized molecules in the process of recombination of polarized atoms from the ABS and the measurement of the nuclear polarization of hydrogen and deuterium molecules is now underway in Jülich, Germany [5]. We proposed a scheme of a setup to demonstrate the possibility to obtain polarized molecules of hydrogen isotopes using the classical Stern-Gerlach method [6]. For this purpose, an available source of polarized deuterium atoms with superconducting sextupole magnets is used [7]. The essence consists in the spatial separation of the molecules in the different quantum states in a strong inhomogeneous magnetic field.

\section{Description of the Experimental Setup}

Fig. 1 shows a photo of two magnets before they are encapsulated in a cryostat. The inner stainless tube of the magnets having a thickness of the wall of $1 \mathrm{~mm}$ determines the available aperture of the magnets of $42 \mathrm{~mm}$ diameter. Two magnets, 7.0 and $12.5 \mathrm{~cm}$ in length, separated by a gap of $31 \mathrm{~cm}$, were used to focus the molecules. The magnetic field at a radius of $21 \mathrm{~mm}$ from the axis of the magnet, with a current in the magnets of $350 \mathrm{~A}$, has a magnitude of $3.4 \mathrm{~T}$ and a field gradient of $320 \mathrm{~T} / \mathrm{m}$. When working with hydrogen, the temperature of liquid helium was lowered to $2.5 \mathrm{~K}$ by reducing its vapor pressure to increase pumping speed for $\mathrm{H}_{2}$. The nozzle was mounted at a distance of $33 \mathrm{~cm}$ from the magnet entrance and could be cooled down to liquid helium temperature. The nozzle is a copper block having a circular slit, with outer and inner diameters of 41.9 and $41.7 \mathrm{~mm}$ (slit width, respectively, is equal to $0.1 \mathrm{~mm}$ ), and its photo is shown in Fig. 2. The nozzle is installed coaxially with the sextupole magnets. The nozzle temperature can be varied over a wide range with the use of a heater. A disk with a diameter of $40 \mathrm{~mm}$ installed at the entrance of the magnets, forming a circular diaphragm with an outer diameter of 42 $\mathrm{mm}$ and a width of $1 \mathrm{~mm}$. The spread of angular velocities at the diaphragm is $\sim 1.5 \cdot 10^{-3}$ radians for molecules that fly from the nozzle along the axis of the magnet. In this geometry, the molecules are passing near the poles of the magnet, where the field gradient is maximal. A disk installed at the entrance to the magnet forms a shadow behind the magnet, where molecules cannot be found with the magnets switched off if they are 
pumped out by the cryogenic surface of the magnet aperture with a probability equal to one. At a distance of $135 \mathrm{~cm}$ from the magnets, this shadow has a diameter of more than $30 \mathrm{~mm}$. Here a compression tube (CT) to receive the molecules $30 \mathrm{~mm}$ in diameter and $20 \mathrm{~cm}$ in length is placed along the axis of the magnets. In such a geometry the direct

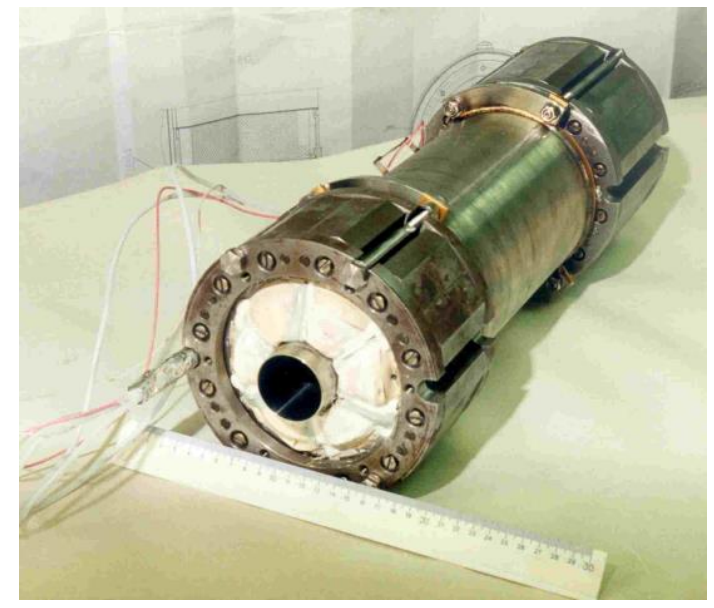

Fig. 1. Photo of superconducting sextupole magnets before they are encapsulated in the cryostat.

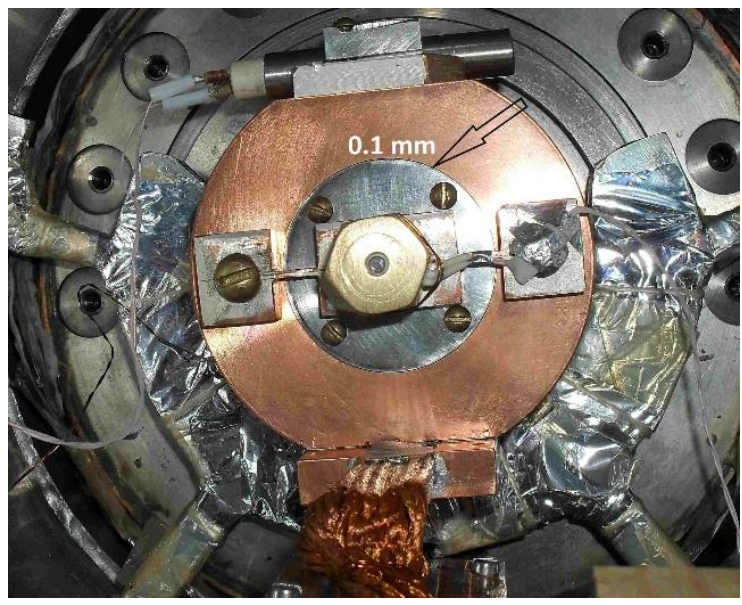

Fig. 2. Photo of the slit nozzle.

ballistic (free motion) flux of molecules cannot reach the CT. Only molecules in the state with $I=1, \mathrm{~m}_{I}=-1$, which are focused by the strong gradient, can enter the CT. A vacuum gauge is installed in the volume of the CT to determine the gas pressure change at the end of the $\mathrm{CT}$ during the magnet ramping. A differential pumping system including two cryopumps was implemented to get an ultra-high vacuum in the vicinity of the CT. The ultimate vacuum in this chamber is below $10^{-9}$ mbar. The chamber that contains the CT could be moved in the transverse direction at a distance of $\pm 6 \mathrm{~cm}$, which allows the measuring of the spatial distribution of the beam intensity at this position. The photograph of the experimental setup is shown in Fig. 3. A more detailed description is given in [8].

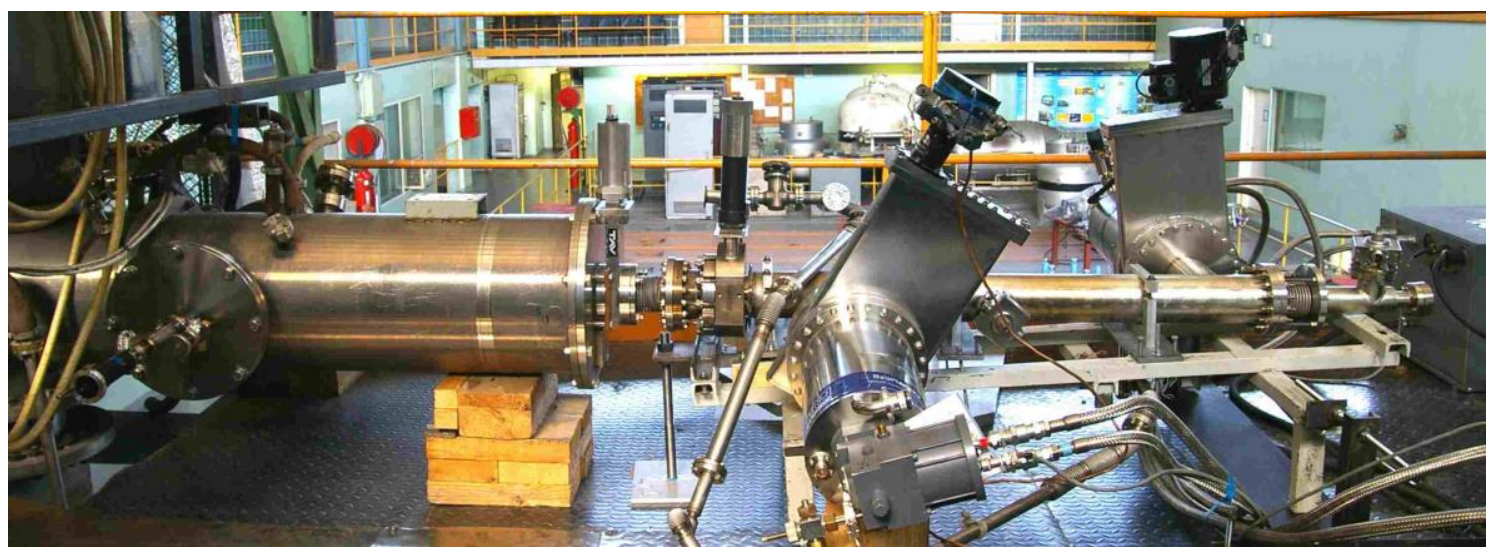

Fig. 3. The photograph of the experimental setup. 


\section{Experimental Results}

Figure 4 shows the experimental result on the pressure change in the CT for a hydrogen molecular beam, when the current through the magnets was turned on and off.

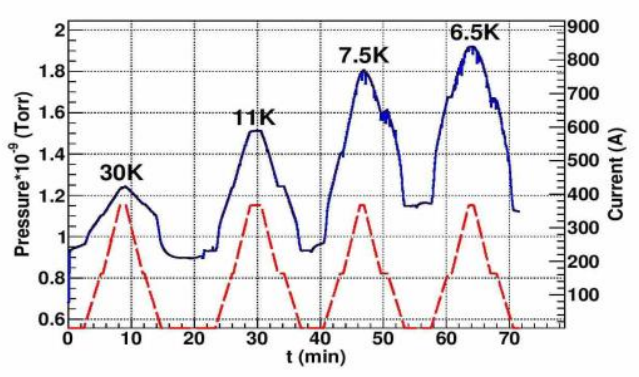

Fig. 4. Dynamics of hydrogen pressure in the receiving tube (solid line) and the current through the windings of the deflecting magnets (dashed curve) with a constant flow of gas through the nozzle. The measurements are performed for different nozzle temperatures.

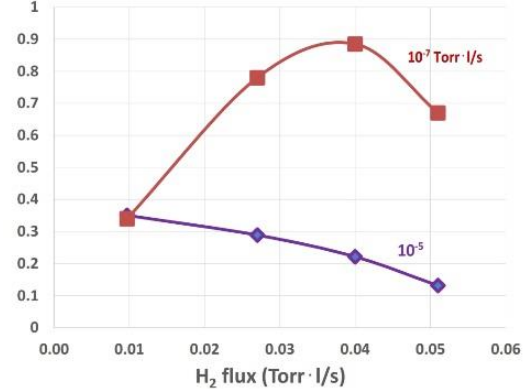

Fig. 5. The intensity of a polarized hydrogen molecular beam entering the compression tube (squares) and the ratio of the polarized molecular flow in the CT to the flux from the nozzle (diamonds), measured vs. the flux from the nozzle.

The measurements have been performed at different temperatures of the nozzle as indicated in Figure 4. If the temperature of the nozzle goes down below $6.5 \mathrm{~K}$ a condensation of gas in the nozzle occurs. Considering that the conductance of the compression tube and the sensitivity of the vacuum gauge to the given gas is known, it is possible to determine the flux of focused (polarized) molecules into the tube. Fig. 5 presents the intensity of the polarized hydrogen molecular beam entering the CT measured as a function of the flow rate from the nozzle. Also, the ratio of the polarized molecular flux in the CT to the flux from the nozzle is given in the same figure. These measurements were performed at 7.3 K nozzle temperature. The maximum flux of the polarized hydrogen molecules focused by the magnets into the CT was measured to be $3 \cdot 10^{12} \mathrm{~mol} / \mathrm{s}$ at a flow rate $4 \cdot 10^{-2}$ Torr $1 / \mathrm{s}$. For higher flow, the intensity of focused molecules is decreased. One probable explanation of this phenomenon is attenuation of the beam by the residual gas between the nozzle and the diaphragm. For collisions of the molecules at so small relative energies, which are realized in our source, there is no experimental data for the elastic $\mathrm{H}_{2}-\mathrm{H}_{2}$ scattering cross section. Theoretical prediction indicate that the cross section may increase to one order of magnitude or more for small energy [9]. The other reasons for the decreasing of the intensity of the beam are probable dimers and clusters production in the beam when the pressure rises. The increased pressure in the nozzle may change the directivity of the angular distribution of the molecules from the nozzle and increases the area from which the free molecular flow begins, comparing with the nozzle size. The ratio of the focused molecules to the total flux from the nozzle was measured to be in the range of $(1.3-3.5) \cdot 10^{-6}$ and it decreases when the flow rate is increasing. The measured ratio is in agreement with the predicted by Monte Carlo simulation, which is $2.3 \cdot 10^{-6}$. For deuterium 
the temperature of the nozzle was raised to $11 \mathrm{~K}$. The flux of the focused deuterium molecules is lower, since the magnetic moments of the deuterium molecules are much smaller than those of hydrogen molecules. In addition, for deuterium molecules that have the same spin projection, the magnetic moments are very different. The measured flux of polarized deuterium molecules is about 7 times smaller than the flux of hydrogen molecules. Note that the geometry of the source was not optimized to get the highest flow of polarized deuterium molecules.

A noticeable level of the signal of unpolarized molecules, which is not predicted by Monte Carlo modelling, is perhaps due to the reflection of molecules from the walls of the vacuum chamber of the magnets. When the angle of incidence, measured from the normal to the surface, is close to $90^{\circ}$, specular reflection may occur, which leads to the appearance of the beam intensity into the CT when the magnets are off.

\section{Conclusion}

In conclusion, as shown by this research, with the already available magnets (not optimal for the given problem), it is possible to produce $3 \cdot 10^{12} \mathrm{~mol} / \mathrm{s}$ of nuclear spin polarized molecules. The obtained results can be used as a basis for the development of a highintensity source of polarized molecules [10]. This source should contain a superconducting multipole magnet with an aperture of one order of magnitude larger than the used one. The magnetic pole tip field should be higher due to an application of a novel type of superconductor. The length of the deflecting field is almost arbitrary and should be optimized to get a proper separation of molecules. Capillaries can be used in the source to get a more directional beam from the nozzle. Polarized molecules could be condensed by cryosurface. It is expected that such a source could provide an intensity of polarized molecules compared with the intensity of the best atomic beam sources.

In the frame of joint RSF and DFG grants it is proposed to measure the degree of polarization of the protons and deuterons in a focused molecular beam using a Lamb-shift polarimeter similar to that used in [5]. In the meantime, the polarimeter has arrived to Novosibirsk from Jülich and the measurements of the nuclear polarization in the molecules will be done soon. The proposed experimental setup is shown in Fig.6.

\section{POLARIZED MOLECULAR SOURCE IAMB-SHIFT POLARIMETER}

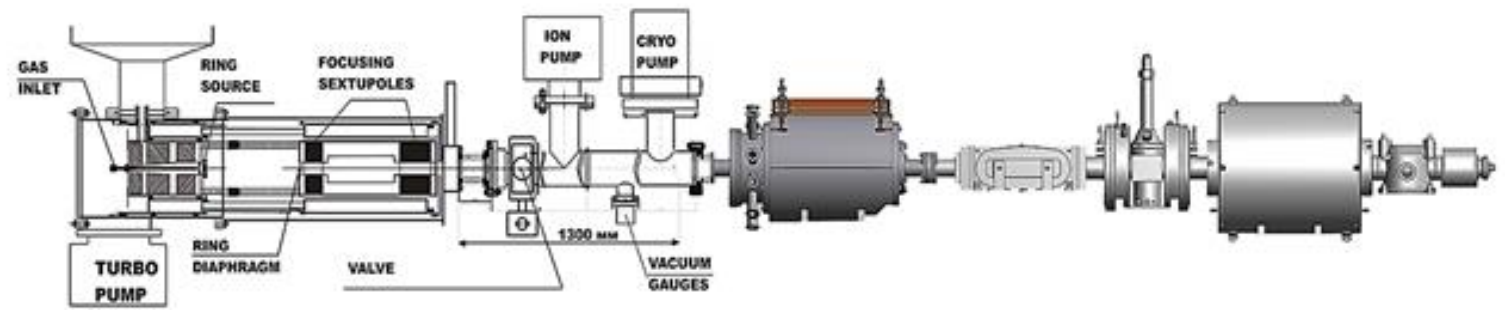

Fig. 6. Molecular beam source and LambShift polarimeter.

The understanding of the polarization losses of the molecules, e.g. due to interactions of the nuclear spin with the rotational magnetic moment, and their suppression may allow the creation of more dense polarized targets. Such targets are on demand for nuclear physics experiments at storage rings of charged particles. Since the deuterium main state 
at low temperatures is ortho-deuterium, probably it is possible to produce solid polarized deuterium and use it as a fuel in inertial fusion reactions and other studies. For more information see $[11,12,13]$.

\section{Acknowledgements}

This work was supported by the Russian Science Foundation under the joint with Deutsche Forschungsgemeinschaft grants RSF 16-42-01009 and DFG BU 2227/1-1.

\section{References}

[1] E. Steffens and W. Haeberli. Rep. Prog. Phys. 66 (2003) 1887.

[2] D. Toporkov, in XVth Inter. Workshop on Polarized Sources, Targets, and Polarimetry. Charlottesville, Virginia, USA, (2013) p. 064.

[3] G. Ciullo, R. Engels, M. Büscher, A. Vasilyev. Springer Proc. in Phys. 187 (2016) 1.

[4] Ch. Leemann, H. Burgisser, P. Huber, U. Rohrer, H. Paetz gen.Schieck, F. Seiler, Helv. Phys. Acta. 44 (1971) 141.

[5] R. Engels et al.; Phys. Rev. Lett. 115 (2015) 113007.

[6] R. Frisch, O. Stern. Z. Phys. 85 (1933) 4.

[7] L.G. Isaeva, B.A. Lazarenko, S.I. Mishnev, D.M. Nikolenko, S.G. Popov, I.A. Rachek, Yu.V. Shestakov, D.K. Toporkov, D.K. Vesnovsky and S.A. Zevakov. Nucl. Instrum. and Methods, A 411 (1998) 201.

[8] D.K. Toporkov, A.V. Gramolin, D.M. Nikolenko, I.A. Rachek, R.Sh. Sadykov, Yu.V. Shestakov, A.V. Yurchenko, S.A. Zevakov. Nucl. Instrum. and Methods A 868 (2017) 15.

[9] Teck-Ghee Lee et al.; J. Chem.Phys. 125 (2006) 11430.

[10] A.V. Yurchenko, D.M. Nikolenko, I.A. Rachek, Yu.V. Shestakov, D.K. Toporkov, A.V. Zorin J. Phys.: Conf. Ser. 938 (2017) 012023.

[11] R. Engels et al.; contribution to this proceedings, POS(SPIN2018) 099.

[12] P. Kravchenko et al.; contribution to this proceedings, POS(SPIN2018) 177.

[13] M. Büscher et al.; contribution to this proceedings, POS(SPIN2018) 180. 